\title{
„Unverlöschliche Merkmale menschlichen Willens" Heinrich Wuttke und die Geburt der Schrift aus dem Geist der Tätowierung
}

\author{
ULRIKE LANDFESTER \\ Universität St. Gallen
}

1872 erschien in Leipzig der erste Band einer, so der Titel, Geschichte der Schrift und des Schriftthums von den ersten Anfängen des Schreibens in der.Tatuirung bis zur Legüng elektromagnetischer Dräthe: Sein Verfasser, der Historiker Johann Karl Heinrich Wuttke, legte damit den ersten Teil eines Lebenswerkes vor, das er vor seinem Tod 1876 nicht mehr vollenden konnte; der erste Band umfasst ,nur' die Zeitspanne vom „Zustand der Schriftlosigkeit“ bis hin zur Erfindung des Alphabets. Wuttke geht darin von einer erstaunlichen Leitbehauptung aus: „Vom Bemalen des Leibes," also dem letzten Stadium der Schriftlosigkeit, ,ward nun der Übergang gemacht zum Einbrennen, Einschneiden, Einätzen solcher etwas vorstellenden Zeichen in den Leib, damit sie unverlöschliche Merkmale menschlichen Willens und Gedankens blieben, zeitlebens dem Körper eingegraben seien" ${ }^{" 1}$ - die früheste Form der Schrift ist die Tätowierung.

Der vielleicht bemerkenswerteste Aspekt dieser Formulierung ist die Tatsache, dass Wuttke hier weder von der Formgeschichte ,solcher etwas vorstellenden Zeichen" aus noch auch im strengen Sinn chronologisch argumentiert. Das Belegmaterial für die Bedeutung der Tätowierung für die Schrift stammt fast ausschließlich aus Quellen des 18. und 19. Jahrhunderts, während die Quellen etwa für die Abschnitte über Keil- oder Runenschrift sehr viel weiter zurückgehen. Ausschlaggebend für Wuttkes Überzeugung von der kathartischen Funktion der Tätowierung in der Geschichte der Schrift ist die Beständigkeit, die das Zeichen erlangt, wenn es als ,unverlöschliche[s] Merkmal[ ] menschlichen Willens" in ein Trägermaterial eingetragen wird, das es ,zeitlebens“ bewahrt. Daran allerdings bricht ein logisches Problem auf, das von Wuttke selbst nicht thematisiert, geschweige denn gelöst wird: Der menschliche Körper und insbesondere seine Haut ist bekanntlich ein Trägermaterial mit einer relativ kurzen Verfallszeit, kürzer jedenfalls als die der Materialien - Holz, Stein, Keramik -, in die der Mensch vor der Schrift seine Bilder einträgt. Der Umstand, dass die Tätowierung in dieser fragilen Subs- 
$\operatorname{tanz}$,zeitlebens eingegraben“ bleibt, ist dennoch in Wuttkes Augen entscheidend dafür, dass an dieser und keiner anderen Substanz das Bild in die Schrift umschlägt. Dieses Paradigma der Unauslöschlichkeit als Angelpunkt dieses Umschlags bezieht demnach sein kulturstiftendes Potential offenkundig aus einem anderen Bedeutungsreservoir als dem der physischen Beständigkeit tätowierter Texte - und dieses Reservoir muss genügend Gewicht besitzen, die Tätowierung gegen den Mangel historischer Evidenz für ihre Positionierung zwischen Bild und Schrift sowie gegen die Vergänglichkeit ihres Trägermaterials als Ursprung der Schriftkultur schlechthin plausibel zu machen.

Dieses Bedeutungsreservoir - so die Kernthese der folgenden Ausführungen - ist das Wissen um die unauslöschlichen Auswirkungen der Schrift auf den menschlichen Körper - ein Wissen allerdings, das von Wuttke nicht ausbuchstabiert wird, sondern seiner Argumentation gleichsam als verborgene Tiefendimension zugrunde liegt. Es lässt sich nicht entscheiden, ob Wuttke diese Bedeutungsschicht seinem Text bewusst unterlegt hat; sicher ist nur, dass sie in verblüffender Genauigkeit dem Entwicklungsstand entspricht, den der europäische Diskurs über die Tätowierung im 19. Jahrhundert erreicht hatte, und dies nicht nur auf inhaltlicher Ebene, sondern auch und gerade im Mangel jeder theoretischen Systematisierung: Wie in Wuttkes Geschichte der Schrift, so ist die strukturelle Affinität von Schrift und Tätowierung auch der Geschichte des Diskurses über die Tätowierung zwar implizit, dabei aber als zentrales Dispositiv der vorgenommenen Zusammenhangsbildungen eingeschrieben.

I.

Der 1818 geborene Johann Karl Heinrich Wuttke gehört zu den produktivsten und zugleich umstrittensten Historikern des 19. Jahrhunderts, nicht zuletzt deshalb, weil seine berufliche Laufbahn von seiner Studienzeit in der schlesischen Heimatstadt Breslau 1835-38 an untrennbar mit seinen intensiven politischen Aktivitäten verbunden war: Eng befreundet mit Hoffmann von Fallersleben, Robert Blum - mit letzterem zusammen gründete er in Leipzig 1845 den Verein für Rhetorik - und später Ferdinand de Lassalle, war er 1848 erst Mitglied des Frankfurter Vorparlaments auf der Seite der gemäßigten Linken; als Blum 1848 in Wien als revolutionärer Aufrührer standrechtlich erschossen wurde, gelangte er als Ersatzmann für diesen als reguläres Mitglied in das Paulskirchenparlament, wo er sich massiv, wenn auch erfolglos gegen die Übertragung der erblichen Kaiserwürde an Preußen einsetzte. $\mathrm{Zu}$ seinen akademischen Lehrern gehörte Leopold Ranke, bei dem er 1839/40 in Berlin studierte, und vor allem Kurt Wachsmuth, als dessen Schüler er sich 1841 an der Universität Leipzig habilitierte. Wuttkes zahlreiche Publikationen dokumentieren ein breites Interessenspektrum, werden heute aber kaum noch gelesen; das einzige Werk, das sich neben seiner Geschichte der Schrift nach 
wie vor einer gewissen Bekanntheit erfreut, ist eine Streitschrift gegen das nachrevolutionäre Zensurwesen von 1866, Die deutschen Zeitschriften und die Entstehung der öffentlichen Meinung in Deutschland. ${ }^{2}$

Als Wuttke Ostern 1841 als Privatdozent in Leipzig seine Lehrtätigkeit aufnahm - 1848 erhielt er dort die Professur für historische Hilfswissenschaften -, begannen seine Vorlesungen nicht zuletzt deswegen sofort eine enorme Strahlkraft zu entfalten, weil er sich gegen die konservativ-dogmatische Geschichtswissenschaft emphatisch als Kulturhistoriker verstand und daher auch mit Quellenmaterialien und Fragestellungen arbeitete, die weit über das eng abgesteckte disziplinäre Feld seiner Zeit hinausgingen. Einer der Gegenstände dieser programmatischen Grenzgänge war schon zu einem sehr frühen Zeitpunkt die Tätowierung: „Meine Auffassung des Tatuirens z.B.“”, so bemerkt Wuttke in der Vorrede zu seiner Geschichte der Schrift, „habe ich zuerst im Jahre 1843 und dann wiederholt in meinen Vorlesungen auf einer so großen Universität wie Lëipzig vor zahireichen Zuhörern ausgesprochen." (VI)

Dieser Vorrede zufolge bildete die Arbeit an der Geschichte der Schrift seit Beginn seiner wissenschaftlichen Tätigkeit deren Gravitationszentrum. Welche Bedeutung gerade die „Auffassung des Tatuirens“ für Wuttke darin besaß, erhellt schon daraus, dass er von der salvatorischen Generalklausel, mit der er die historiographische Integrität seiner Ausführungen behauptet - „Was in diesem Buche von den herrschenden Schulmeinungen abweicht, hat sich mir aufgedrungen," ist also nicht das Resultat einer bewussten Deutungsanstrengung - allein das Feld der Tätowierung ausnimmt:

Einzig und allein hinsichtlich der Tatuirung habe ich gesucht, die Ansicht zu erweisen, die ich im Jahre 1840 oder 1841 aus Gesprächen mit dem Staatsrathe Tỉlesius von Tilenau, dem Leiter der wissenschaftlichen Abtheeiliüng der Krusensternschen Weltumsegelung in den Jahren 1803-1806, in Spaziergängen im Rosenthale mir gebildet hatte. (VIII)

Tilesius hatte während der Weltumsegelung den Großteil der Illustrationen gezeichnet, die dann, in Kupfer gestochen, mit deren Dokumentation 1814 veröffentlicht wurden, ${ }^{3}$ darunter das Porträt eines ganzkörpertätowierten Mannes aus Nukahiva, das Wuttke auch in den Tafelteil seines Buchs aufnahm.

Zweierlei ist an diesem Abschnitt der Vorrede auffällig. Erstens wird hier eine Geschichte der Geschichte des eigenen Schreibens skizziert, an deren Ursprung ebenfalls die Tätowierung steht, so dass die nachfolgende Historiographie einen entschieden selbstreferenziellen Bezugshorizont erhält. Zweitens und wichtiger noch - geht ihm unmittelbar eine Apologie der von Wuttke ,nur zagend" getroffenen „Anordnung des Stoffes [ . . . ] in diesem Bande" (VII) voraus. Hier gibt der Verfasser offen zu, dass er bei dessen Organisation ,die Zeitfolge verließ, " und rechtfertigt die Konstruktion, mit der er das Material in den Dienst seiner Idee stellt, damit, dass ,die Stufenfolge der Erscheinungen die Einsicht in den allgemeinen Gang befördert" (VIIf.). Dieses vordergründig 


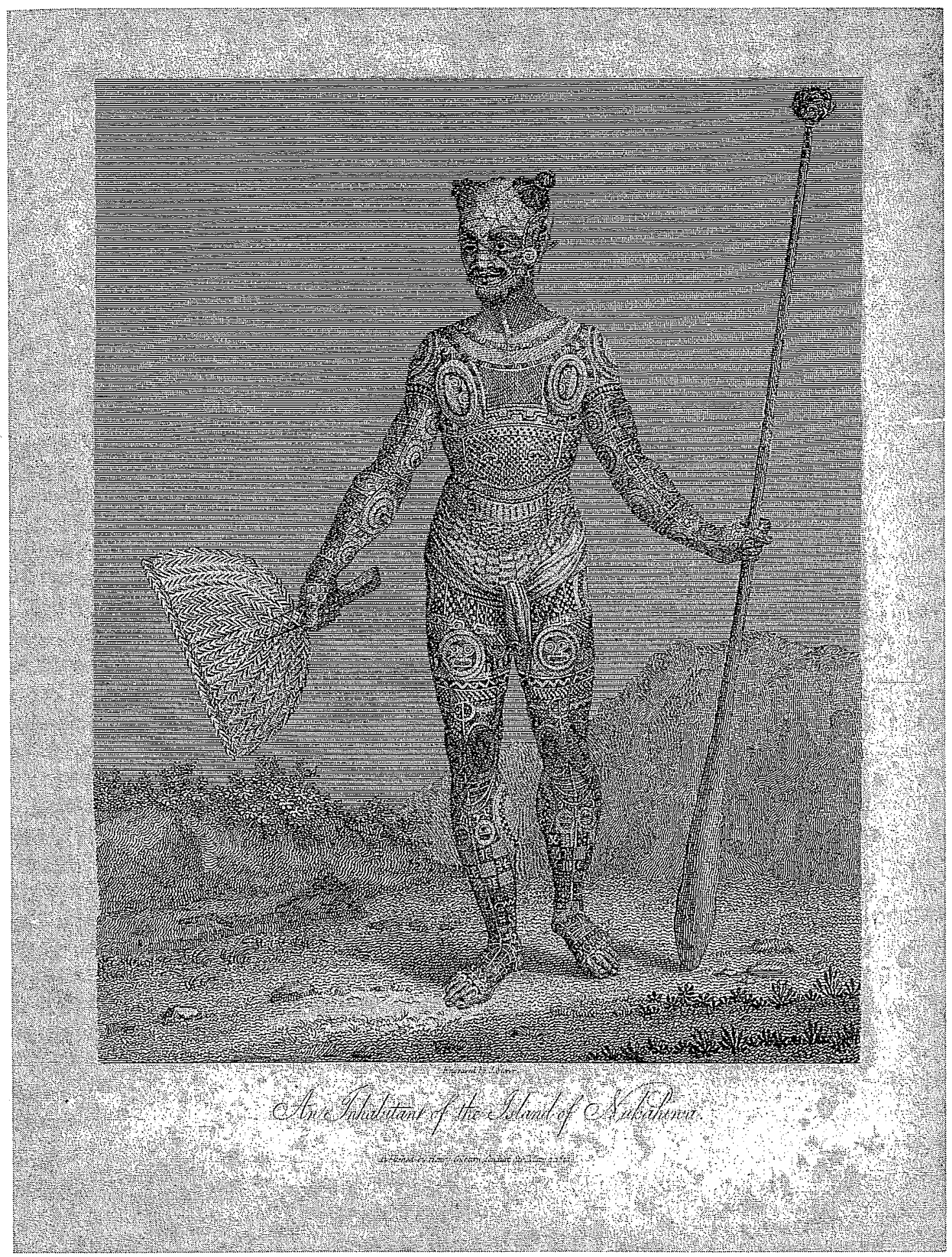

Ein Bewohner der Insel Nukahiva / An Inhabitant of the Island of Nukahiva; aus: Georg Heinrich von Langsdorff, Voyages and travels in various parts of the world, during the years 1803,1804, 1805, 1806, and 1807. Illustrated by engravings from original drawings (London: Colburn, 1813-14) 117. (Wisconsin Historical Society Library Rare Book Collection) 
didaktische Argument ist faktisch ein Bekenntnis zu einem Geschichtsbild, das die synchrone Koexistenz von körperbemalenden Wilden, tätowierenden Halbzivilisierten und schreibenden ,richtigen' Menschen als einen Zustand der Koexistenz verschiedener menschheitlicher Entwicklungsstadien deutet und auf dieser Basis von der Gegenwart aus die Chronologie einer in sich linear verlaufenden Schriftgenese zu extrapolieren erlaubt.

Während die Vorrede den Konstruktionscharakter dieses Geschichtsbildes immerhin noch andeutet, entwerfen Wuttkes „Einleitende Bemerkungen“ dieses Bild dann bereits als Gewissheit:

Anfänglich mussten die Menschen, um ihres Unterhalts willen auseinandergehen. Aus ihrer Zerstreuung über den weiten Raum der bewohnbaren Erde ergaben sich nothwendig getrennte Entwickelungen. Verschiedene Völker entstanden: Abtheilungen des Menschengeschlechtes machten Völker aus, die in sich lebten. Und indem das Wort in der Mannichfaltigkeit seines Erscheinens zu einer Vielfältigkeit der Sprächen wurde, war mit jeder verschiedenen Sprache ein abschließender Kreis gezogen, innerhalb dessen allein die enge Verbindung des Zusammenwachsens bestand. So war also das erste ein Zerfall der Menschheit in gesonderte Theile. Die Bildungen arteten ungleich, die Völker entfalteten sich mit verschiedener Schnelligkeit und auf verschiedene Weise. (7)

Diese geschichtsphilosophische Grundannahme wird von den eigentlichen Ausführungen des Buches in einer eigentümlichen Spannung zwischen typologischen und geographisch fokussierten Darstellungskriterien umgesetzt: Auf ein Kapitel zum Zustand der Schriftlosigkeit folgt eines zu den Vorstufen der Schrift und dann ein weiteres zu den Anfängen der Schrift mit der Tätowierung, das durch zwei kurze Abschnitte zu Gegenständen und Zeichnungen ergänzt wird. Hier schließt eine Serie von einzelnen Ländern gewidmeten Kapiteln an - Nordamerika, Südamerika, Peru, Mittelamerika, Chină, Koreá, Japan, Hinterindien und die Mongolei, Ägypten, Südwestasien; die letzten beiden Kapitel gelten den Sprachlauten und dem Alphabet.

Die typologische Rahmung des länderspezifischen Mittelteils erscheint auf den ersten Blick kontraintuitiv, umso mehr, als die Schriftgeschichte Europas im Mittelteil fehlt, während sie einen Großteil des in den typologischen Kapiteln ausgewerteten Materials beistellt. Auf den zweiten Blick aber realisiert diese Rahmung performativ genau die Konsequenzen, die sich aus Wuttkes Geschichtsbild für die Darstellung der Schriftgeschichte ergeben: Der erste Teil von der Schriftlosigkeit bis zu den Anfängen der Schrift übersetzt die Annahme von der Gleichursprünglichkeit der Menschen in ein Argument für die strukturelle Gleichursprünglichkeit der Schrift, behauptet also, dass der Übergang zur Schrift unabhängig von lokalen Varianzen prinzipiell immer gleich verläuft. Der Mittelteil verhandelt auf dieser Basis die einzelnen Verlaufsgeschichten der Schrift nach dem ,Zerfall der Menschheit in gesonderte Theile" (7), während die Erfindung des Alphabets aus den Sprachlauten dann 
abschließend - von Wuttke mehrfach explizit so erklärt - den ersten großen Schritt auf eine Reintegration der Menschheit hin vollzieht: Und dann ist es schon nicht mehr verwunderlich, dass die europäische Schriftgeschichte nicht als eigenes Kapitel geschrieben werden muss, ist sie doch als Ursprung und Schauplatz des alphabetarischen Schreibens omnipräsente Möglichkeitsbedingung und zugleich Fluchtpunkt des gesamten Projekts.

\section{II.}

In diesem Sinne ist Wuttkes Geschichte der Schrift eine Darstellung von erheblichem rhetorischem Kunstcharakter und einer konsequent performativ realisierten Inszenierungslogik. So beruht die Idee von der strukturellen Gleichursprünglichkeit der Schrift auf einer von Wuttke in den „Einleitenden Bemerkungen" kursorisch gesetzten Denkfigur, derjenigen nämlich von der Gleichursprünglichkeit von Schrift und Bild: „Im Anfang stand Malen und Schreiben zusammen, verbunden als eins.“ (16) Erst die „Lostrennung von Schrift und von Malerei" (ebd., Hvh. im Original) ermöglicht die Ausbildung des Abstraktionsgrades, mit dem erstere zu sich, also zu ihrer spezifischen Zeichenhaftigkeit kommt:

Dann haben wir eine Gemäldeschrift vor uns, die mittelst Zeichnung von sichtbaren Gegenständen die Gedanken gibt, ohne deren sprachliche Darstellung zu enthalten. Dieser bloße Vorstellungen mittheilenden Satzschrift folgt eine Wortschrift, welche unmittelbar keine Gedanken, sondern zunächst nur Worte bietet, nur Worte malend zum Auge spricht [ ... ]. Hernach tritt die Sylbenschrift, zuletzt die Alfabetschrift auf [ ... ]. Diese kann nicht, gleich den Darstellungen der Dinge, allgemein verständlich sprechen, ist nur für eine bestimmte Sprache lesbar. Auch treten bei ihr an die Stelle der Żeichnungen bestimmte Zeichen. [ ... ] Nachdem das Alfabet einmal vorhanden war, eigneten viele Völker es sich an [ ... ]. (Ebd.)

In diesem letzten Stadium scheint vorübergehend ein Zustand der Reintegration erreicht; da aber die Völker jeweils ihre eigene Sprache alphabetarisch notieren, zerfällt die Einheit gleich wieder, auch wenn sie in der Zeichenstruktur als solcher zumindest als Versprechen einer zukünftigen Wiedervereinigung der Menschheit erhalten bleibt.

Mit seinem ebenso emphatischen wie weit gefassten Schriftbegriff, dessen wichtigstes Charakteristikum eine ungewöhnlich scharfsichtige Wahrnehmung der Bedeutung des Schreibprozesses und der darin verwendeten Medien für die Geschichte der Schrift ist, kann Wuttke durchaus als einer der ersten großen - und als solcher zu Unrecht vergessenen - Kulturtheoretiker der modernen Schreibszene gelten. ${ }^{4}$ Die Basis dieses Schriftbegriffs ist instrumentalistisch, die für den Gegenstand selbst aus diesem Begriff resultierenden systematischen Effekte aber betonen vor allem seine performative Dimension: 
„Eine von den großen Aufgaben, welche das Menschengeschlecht zu lösen hat, besteht darin, die Macht des Menschen über das Maß seiner natürlichen Ausstattung hinaus zu erhöhen, " so der Beginn der „Einleitenden Betrachtungen“: „In einer Hauptrichtung ist ihm dies gelungen durch die Erfindung der Schrift und ihrer Steigerung im Druck, in der Stenografie und in der elektromagnetischen Telegrafie, und zwar gelungen in einem Grade, welcher, so weit wir heute zu sehen im Stande sind, die nicht überschreitbare Grenze beinahe schon erreicht." (1)

Das Instrument Schrift ist also Wuttke zufolge zweifach determiniert, einmal über seine Speicherfunktion, zum anderen, darauf aufruhend, als Kommunikationsformat, das die Grenzen der Gegenwart sowohl zeitlich als auch räumlich überschreiten kann, und dies umso effizienter, je weiter die technische Entwicklung der Aufschreibesysteme ${ }^{5}$ fortschreitet: „Das Schreiben selbst beschleunigt Malling-Hansens. Maschine“- - die 1865 von dem gleichnamigen dänischen Ptarrer patentierte Schreibkugel - ,und die Telegrafie hat in die Ferne Zeichen zu geben vermocht. Endlich hat die große Erfindung unseres Jahrhunderts, der elektro-magnetische Telegraf, gelehrt Gedanken mit Blitzesschnelle über Länder und Meere hinweg zu tragen." (10) Aufgrund dieser Effizienz ist die Schrift das ideale Medium dafür, die verlorene Einheit der Menschen als Einheit des „Schrifttums“ zu restituieren:

Wie die Schrift, weil sie die trennenden Zwischenräume unter den Menschen aufhebt, damit die Abwesenheit in Anwesenheit verwandelt und Vergangenheit zur Gegenwart macht, so bildet alles was in ihr niedergelegt und so aufbewahrt worden ist, den jederzeit verwendbaren und dennoch allezeit beständigen $G e$ sammtbesitz des Menschengeschlechts und zwar denjenigen, welchen sich dieses selber erzeugt hat. (15)

Was Wuttke nicht auszusprechen für nötig hält, ist, dass freilich nur Schriftkundige an diesem Besitz partizipieren können und damit auch nur sie dem „Menschengeschlecht“" angehören. Aber was hier zunächst den bösen Charme "elitären Kulturchauvinismus' zu verströmen scheint, wird zumindest im Rahmen seiner Schriftgeschichte dadurch relativiert, dass für Wuttke der Wille zur Schrift ohnehin ausnahmslos jedem Menschen immanent ist, gleich, ob er früher oder später in deren Erfindung einmündet. Deshalb kann er seine Geschichtsdarstellung mit den typologischen Kapiteln zum Beginn der Schriftkultur einsetzen lassen. Wuttkes Schriftbegriff bezieht seine Emphase also aus einer programmatischen Verkoppelung von Menschsein und Schrift, derzufolge allerdings der in der Schriftkultur weiter fortgeschrittene Mensch stets der bessere, ,menschlichere' ist. Für den Gegenstand seiner Darstellung hat dies zwei Konsequenzen: Erstens privilegiert die Ausweitung seines Schriftbegriffs auf die elektromagnetische Telegraphie die technologische Komponente der Schrift zuungunsten der ästhetischen Komponente, die denn auch in seinem Buch allenfalls als besondere Form der Mnemotechnik erscheint. Zwei- 
tens gerät die Schrift in dem Maß, in dem sie vom Instrument des Menschen zum eigentlichen Humanum aufgewertet wird, vom Gegenstand einer historiographischen Deskription zu einem als Telos der Menschheitsgeschichte hoch besetzten Objekt der Begierde, das es in seiner richtigen Form überhaupt erst $\mathrm{zu}$ konstituieren gilt.

Gegenüber diesem starken Schriftbegriff bleibt der Bildbegriff Wuttkes vergleichsweise unscharf, zumal er sein Gewicht ausschließlich aus der Schrift bezieht. Im vorschriftlichen Zeitalter kennt der Mensch auch keine Bilder; das entsprechende Kapitel entfaltet den Zustand der Schriftlosigkeit vielmehr zwischen den beiden Figuren des Tiers und des Schmerzes. Tertium comparationis ist der mnemotechnische Aspekt der noch nicht existierenden Schrift: Tierhaft ist der schriftlose Mensch, weil er nicht zu erinnern bzw. das Erinnerte nicht festzuhalten vermág und deshalb auf die Gegenwart angewiesen bieibt. Erstes Anzeichen einer beginnenden Emanzipation ist die mündliche Poesie, also die gebundene Rede, in der Reim und Metrum als Gedächtnisstützen fungieren, und dann die Erfindung der performativen Rede etwa in Rechtsformeln; ,in derartigen Redensarten (mehr war's im Grunde nicht) pflanzte sich die Rechtskenntniß in Rom fort" (57), bemerkt Wuttke dazu abschätzig, während man in Deutschland die demgegenüber bereits geradezu fortschrittliche Praxis der Koppelung von Schmerz und Erinnerung ${ }^{6}$ pflegte:

Bei wichtigen Anlässen, als z.B. der Setzung eines Grenzsteins zwischen Feldmarken, wurde eine Anzahl Knaben versammelt, damit noch in ferner Zeit Zeugen des Vorganges am Leben seien, und auf dass die Kinder auch gehörig daran dächten, wurden sie auf die neugesetzten Steine gestaucht, unversehens mit den Köpfen aneinander gestoßen und endlich tüchtig an den Ohren gerissen oder genhrfeigt. (58)

Ähnlich bildlos operiert das Kapitel „Vorstufen“. Es setzt mit Verfahren der Zuschreibung von Bedeutung an Gegenstände ein - etwa der der Regierungsgewalt an einen Stab - und geht dann auf dem Weg über das Zeichen wie den aus einem Türpfosten herausgeschnittenen Span als Besitzzeichen pars pro toto für das gesamte Haus zur Zeichenbildung durch Einschneiden etwa von Zählkerben in ein Holzstück über. Es suggeriert also eine Verschiebung dessen, was das Zeichen als solches konstituiert, vom Gegenstand an sich auf den Gegenstand als Trägermedium, und gelangt von hier aus zur Besitzmarkierung von Gegenständen und vor allem Tieren durch eingeschnittene oder eingebrannte Signaturen, deren äußere Form nicht thematisiert wird. Diese Signaturen werden dann ihrerseits zu einem Zeichen, das des mit ihm markierten Gegenstandes nicht mehr bedarf, um wirksam zu sein, sondern vielmehr als Markierung stets auf die Identität seines Besitzers verweist; „Solche Zeichen $[\ldots]$ vertraten, ihrem Sinn entsprechend, die Unterschrift des Namens." (65) Die konsequente Steigerung der Identitätsmarke ist das magische Zeichen, konkret der zauberische Knoten, der in der Lebenswelt des 
Menschen die Gegenwart übersinnlicher Mächte beschwört, gleichsam also als deren Signatur.

Die zweite der beiden Vorstufen und als solche eine Steigerungsform der ,Gegenstände als Mahner' ist die Hautmalerei. Im Gegensatz zu den vorigen Abschnitten ist hier tatsächlich von Bildzeichen die Rede; Wuttke führt eine Fülle von Figuren und Ornamenten auf, die die Körper der verschiedensten Völker zieren. Der Formsprache dieser Bilder aber widmet er kaum eine Randbemerkung; was ihn interessiert, sind ausschließlich die unterschiedlichen Motive für die Hautmalerei, etwa das Bedürfnis nach Verschönerung oder hygienische Gründe, sowie die Frage, ob der Körper immer oder nur zu bestimmten Anlässen bemalt wird - letzteres bezeugt eine höhere Kulturstufe als ersteres - und worin diese Anlässe bestehen. Daraus zieht Wuttke zwei Befunde, deren Bedeutung nicht so sehr das Phänomen der Hautmalerei selbst betrifft als vielmehr das für Wuttkes Argumentation entscheidende Kapitel zur Tätowierung strükturell vorbereiten: Zum einen diagnostiziert er, dass die „Art des Bemalens [... ] keineswegs ganz und gar in jedermanns Willkür“ (76) stand, dass es sich also bei der Hautmalerei um einen konventionalisierten Code handelte, der damit vom folgenden Kapitel aus den Status einer Vorstufe zur Tätowierung beanspruchen kann. Zweitens konstatiert Wuttke, dass, ,wenn rohe, sehr niedrigstehende Völker in Färbungen und bunte Figuren eine Bedeutung hineintrugen, diese Bedeutung im Zusammenhange mit abergläubischen Vorstellungen stand" (ebd.), dass also Bedeutung nicht nur als kommunikativ-pragmatischer, sondern auch und vor allem als intrinsisch metaphysischer Mehrwert eines Zeichens konstituiert worden sei.

Im Zusammenhang von Wuttkes Geschichte der Schrift erfüllt die Geschichte der Hautmalerei die Funktion einer Gelenkstelle, an der ein eigen-. tümliches Dreiecksverhältnis zwischen Schrift, Bild und Körper entsteht: Der Mangel an auch nur spekulativen Bildreferenzen für die ,Gegenstände als Mahner', deren spezifisches Design spätestens bei Brandmarkung und Siegelung für Stoßrichtung und juristisches Gewicht solcher ,Mahnung' historisch eine erhebliche Rolle spielt, korrespondiert mit einer Distanz zwischen Zeichen und menschlichem Körper, die durch die Koppelung von Schmerz und Erinnerung, durch das germanische Ohrenreißen etwa, eher unterstrichen als relativiert wird, hinterlassen derartige Techniken doch keinerlei äußerlich lesbare Spuren auf dem Trägermedium der solcherart eingetragenen Erinnerung. Erst als diese Distanz schwindet, indem die Hautmalerei das Trägermedium Körper und die Herstellung wiedererkennbarer Zeichen einander annähert, erscheint jetzt auch das Bild im Horizont der Darstellung, dessen Bedeutung nun auch das Konnotat von Schmerz und Gewalt in sich aufnimmt: Fasziniert berichtet Wuttke von den Farben und Formen, mit denen insbesondere nordamerikanische Indianer auf Haut und Haaren die von ihnen getöteten Feinde dokumentieren oder die Drohung unbefriedigter Blutrache ausstellen - und beschließt in harter Fügung das Kapitel mit einem Hinweis auf eine der wirk- 
mächtigsten Bildtraditionen des christlichen Abendlandes, auf die Farbsymbolik nämlich, mit der die Maler „,beinahe stets dem wiederauferstandenen Christus ein rothes Kleid und der Madonna einen blauen Mantel“ geben: „[A]uch im Abendlande, auf weit höheren Stufen“ sei „[e]in mystisches Spiel mit den Farben getrieben" (77f.) worden.

Erst in dem Kapitel über die Tätowierung wird das Bild nun explizit auch als Bild Gegenstand von Wuttkes Kulturpoetik der Schrift: Der Mensch habe ,in Bilder, Farben, in manche Verzierungen [ . . . ] einen Sinn hinein[getragen], so daß die Zeichen nicht mehr willkürlich waren" (79), und sei so zur Tätowierung gelangt, die Wuttke gegenüber der Hautmalerei und allen anderen vorschriftlichen Zeichen nobilitiert, indem er ihr den Titel der „Ätzschrift" verleiht - einen Titel, der einen spezifische polemische Pointe "enthält: Wuttke war durchaus bekannt, dass die Farbe, die beim Vorgang deri Tätowierung unter die Haut des Tätowierten eingebracht wurde, nicht per se eine ätzende Flüssigkeit war. Es lag ihm jedoch offensichtlich daran, das Konnotat des Schmerzes bereits in der Überschrift dieses Kapitels deutlich als zentrale Komponente der Tätowierung lesbar zu machen.

Aus diesem Grund auch beginnt das Kapitel selbst nicht direkt mit der eigentlich in seinem Mittelpunkt stehenden Farbtätowierung, sondern mit der von Wuttke als „Mankaverfahren“ (79) bezeichneten Technik der Narbentätowierung. Die Darstellung dieser Technik orientiert sich nicht an den Zeichen selbst, sondern an ihren Auswirkungen auf den Körper, indem sie in ihrer betonten Brutalität sichtlich über das Signalement des von den Tätowierten erfahrenen Schmerzes hinaus auch auf die Schmerzgrenzen des Lesers zielt: Was mit „tiefen Einschnitten, welche Narben zurückliessen“ (80), anfängt, „mit Messern eingeschnitten und die blutende Wunde mit dem Staub einer Pflänze bestreut“" (82) oder, schlimmer, mit Sand, steigert sich geradezu zu Schockeffekten: Da wird „Kafferinnen [ . . . ] ein Pfriem 2 bis 3 Linien unter der Oberhaut durchgestoßen und die Haut über dem Pfriem danach aufgerissen“ (ebd.), da machen die Australier „mit scharfen Muscheln oder Kieseln tiefe Risse in die Haut und halten diese Aufritzungen eine Zeit lang offen, so daß lange starke Narben, fingerdicke Schwielen entstehen" (83) - eine, wie Wuttke abschließend bemerkt, ,ältere und rohere Weise der Hautschrift als das Tättowiren oder Punktiren und Färben, in welchem man eine künstlichere, fortgeschrittenere Behandlungsart erkennt" (86), dieser aber unter dem Aspekt des Schmerzes fundamental artverwandt.

So ist es schließlich der argumentationslogisch an diesen Aspekt gebundene Vorgang des Eingriffs in den menschlichen Körper, an dem das Bild im Abschnitt zur „fortgeschritteneren“ Farbtätowierung als Bild eingeführt wird, um dann endgültig in der Schrift aufgehoben zu werden. Der dafür entscheidende Schritt ist die Fixierung des als Hautmalerei noch flüchtigen Bildes in einem „Beschreibstoff" (17, Hvh. im Original), einem Trägermedium, aus dem das darin eingetragene Zeichen nur durch seine - des 
Trägermediums - Zerstörung herausgelöscht werden kann. Das Differenzkriterium, das Narben- und Farbtätowierung voneinander trennt, ist dessen Oberflächenbeschaffenheit: Die Narbenzeichnung besitzt zwar bereits die Beständigkeit der Tätowierung, noch nicht aber die materielle Diskretion, mit der das Zeichen in seinem Trägermaterial gleichsam substanzlos und doch deutlich sichtbar wird. Die Tätowierungsfarbe wird ja bekanntlich unter die Lederhaut eingebracht, so dass das Zeichen nach Verheilen der Wunde dieselbe Oberflächenstruktur besitzt wie die darüber geschlossene Haut.

In Wuttkes geographisch wie zeitlich weit ausgreifenden Ausführungen zur Farbtätowierung gelangt das Bild zunächst zu einer flamboyanten Blüte. Hier erscheinen im Text viele und ausführliche Bildbeschreibungen, vom einfachen Punktornament über akribisch protokollierte komplizierte Ganzkörpermuster bis zu Tier- und Pflanzendarstellungen, und hier setzt Wuttke auch zu einer Analyse der Formensprache tätowierter Bilder an:

Um wenigstens den Anfang eines Erklärungsversuches zu machen, möchten wir den Vorrath von Tättowirzeichen in zwei Gattungen zerfällen, nämlich in einfache und zusammengesetzte, welche letztere aus der Verbindung mehrerer Zeichen entstanden sind. Weiter möchten wir sie in vier Ordnungen zerlegen, je nachdem die Zeichen 1) Abbildungen von Gegenständen sind, oder 2) Abbildungen von Bäumen, Thieren, oder Theilen des Menschenleibes [ ... ] - oder 3) einfache Striche und Streifen, seien diese gerade, seien sie krumm gezogen, Bänder, Kreise, Spirale, Wellenlinien, oder endlich 4) aus solchen Strichen zusammengezogene Figuren, welche gewöhnliche mathematische Formen oder arabeskenartige Gestalten bilden. (131)

Dieses systematische Sortierung der beschriebenen Zeichen allerdings bleibt ohne Auswertung, mit Ausnahme einer lapidaren Schlussbemerkung:

Eine lebendige Einbildung war allerdings erforderlich, da verständliche Bilder herauszulesen, wo unsere Nüchternheit nur ein Liniengewirr erblickt [ . . . ] Wo die Kunst des Zeichnens ihre unbehülffichen Anfänge erst macht, da ergänzte die unzulänglichenn Umrisse das schauende Auge. (131f.)

Was genau nun das ,schauende Auge“ des Europäers in all seiner „Nüchternheit" aus diesem „Liniengewirr“" herauslas, lässt Wuttkes Text ungeschrieben und das gezielt. Was die Tätowierung in Wuttkes Augen zur Schrift macht, ist, neben ihrer Beständigkeit ihre Unlesbarkeit, da allein diese die Sicherheit garantieren kann, dass ,,in den Tättowirzeichen ein uns verborgener Sinn“ (140) liegt. Weder die mimetische Wirklichkeitsreferenz der pictura noch der ästhetische oder magische Gehalt der imago werden von ihm als Schlüssel für diesen verborgenen Sinn auch nur in Betracht gezogen. Der Bildwert der Tätowierung ist völlig irrelevant, das Bild wird gleichsam aus sich selbst herausgelöscht, um als ein Zeichen zurückzubleiben, das auf alles mögliche verweisen kann, nur nicht auf das, was es allenfalls darstellt. „[D]as Aufzuschreibende,“ so der Schlussabsatz dieses Kapitels, „hing mit der Sprache noch 
nicht unmittelbar zusammen, das Verfahren war ein äußerst langsames und sogar schmerzhaftes, und gleichwol konnte es nur in sehr beschränktem Umfange Anwendung finden und Nutzen schaffen - aber es war doch ein Anfang“" (ebd.) - für die Schrift.

Und doch entgeht auch Wuttkes eigener Text der Macht des Bildes nicht, wenn diese Macht auch die Wiederkehr des Verdrängten nur in dessen vermitteltster Form durchzusetzen vermag, in derjenigen nämlich der Metapher und zwar einer ganz besonderen: Seine „Einleitenden Bemerkungen“ sind durchsetzt mit einer auffallend häufig wiederkehrenden Körpermetaphorik für Sprache und Schrift. „In das Wort hat sich der Geist ergossen; es ist der Leib der geistigen Äußerung, das Fleisch des Gedankens," heißt es da, oder: „Das Wort hatte sich gleichsam potenzirt, als es einen neuen Leib anzog und zur Schrift wurde" (4f.). Wuttke übernimmt hier jenen Gestus, mit dem der Evangelist Johannes die "Geburt Christi als Effekt èines göttlichen Sprechaktes deutet: „Und das Wort ward Fleisch und wohnte unter uns, und wir sahen seine Herrlichkeit, eine Herrlichkeit als des eingeborenen Sohnes vom Vater, voller Gnade und Wahrheit" (Joh. 1.14). Im Licht dieser Metaphorik gewinnt der Übergang zur Schrift an der Tätowierung noch einmal eine neue Dimension: Wenn der menschliche Körper Bezugshorizont für die bildhafte Rede über Sprache und Schrift ist, das Bild aber am menschlichen Körper als Tätowierung den Status der Schrift erwirbt, dann ist nicht eine wo auch immer historisch zu positionierende Stufe, sondern der Körper selbst der Übergang, an dem die Schrift entsteht. Damit aber schlägt der ganze Geschichtsentwurf Wuttkes unversehens von der historiographischen Ordnungsfiktion in die anthropologische Diagnose einer dem Körper des Menschen als Wille zur Schrift a priori immanenten Verbindung zur Schrift um. Diese Verbindung deprivilegiert den ikonischen Chàrakter des Bildes als pictura oder imago von vornherein gegen die am Bild als Zeichen durchgeführte Reflexion auf die Zurichtung des Körpers durch die Schrift - und setzt so die Tätowierung in den Status einer Metapher für die Auswirkungen der modernen Schriftkultur auf den menschlichen Körper ein.

\section{III.}

Die Dimension dieser Zurichtungsbildlichkeit und schon gar ihre Konsequenzen für die Metaphorologie, als die der Zusammenhang von Schrift und Tätowierung von Wuttke inszeniert wird, sind dem Text seines Buches nicht explizit und schon gar nicht programmatisch eingeschrieben, sondern performativer Effekt des Verfahrens, dem Wuttke das von ihm verwendete Material unterwirft. Wuttke nämlich wertet dieses Material durchweg als unzweifelhaft dokumentarisches aus, so, als protokolliere es faktische Realität, ohne dabei in Betracht zu ziehen, in welchem Ausmaß nicht nur die Verschiedenheit der je herangezogenen Textsorten, sondern auch die jeweiligen Standorte ihrer 
Verfasser - ganz zu schweigen von seinem eigenen - diese Realität weniger aufgezeichnet als überhaupt erst hergestellt haben. Das Resultat ist eine Darstellung, die gerade hinsichtlich der Tätowierung ihren Gegenstand weder ethnologisch noch historisch objektiviert. Sie reproduziert vielmehr ein Deutungsmuster, das den europäischen Diskurs über die Tätowierung in Moderne und Vormoderne dominiert hat.

Dieses Muster ist das derjenigen kulturpoetologischen Kippfigur, die Tätowierung und Schrift bereits lange vor Wuttkes Niederschrift seiner $G e$ schichte auf eine wechselseitige metaphorische Affinität angewiesen hat. Sie verdankt ihre Emergenz vor allem der Tatsache, dass die Tätowierung als Artefakt formal nicht a priori auf Bild- oder Schriftcharakter festgelegt ist, als Technik aber eine solche Festlegung verfügbar zu machen scheint: „The minimum definition of tattooing, "so Alfred Gell in seinem Buch über die marquesanische Tätowierung,

the basic technical schema of puncturing the skin and inserting pigment, cannot by itself suffice to delimit any particular symbolic meaning. The age and sex of the tattooing subject, the nature and extent of the designs made, their positioning on the body, the institutional framework of the tattooing process, and many other factors, make all the difference in the world, even within a single 'tattooing system', let alone in cross-cultural perspektive. ${ }^{7}$

Das je tätowierte Motiv ist ein Sekundärphänomen, das räumlich wie zeitlich stark variieren kann und sich demnach nicht einmal von regional oder zeitlich begrenzten, noch weniger aber von global ausgerichteten Versuchen systematischer Auswertung erfassen und schon gar nicht einem an alphabetischen Notationsverfahren erworbenen Schriftbegriff subsumieren lässt. Wuttkès Geschichtsentwurf trägt dieser Unvereinbarkeit insofern Rechñung, als er sich der formtypologischen Komponente der Interferenz von Tätowierung und Schrift so elegant wie konsequent entzieht und stattdessen, ohne ihn allerdings auszubuchstabieren, den verborgenen Subtext einer verfahrenstechnischen Affinitätsunterstellung realisiert. Der Herstellungsprozess des Artefakts Tätowierung durch Punktierung der Haut und Einführung von Farbpigmenten nämlich, der auch von Wuttke in seiner Langwierigkeit und Schmerzhaftigkeit immer wieder hervorgehoben wird, lässt sich doppelt auf den Herstellungsprozess des Artefakts Schrifttum beziehen, einmal durch seine strukturelle Ähnlichkeit mit dem Auftrag von Farbe anhand eines spitzen Instruments auf einen Textträger, zum anderen durch die von Wuttke ebenfalls wiederholt betonte Bedeutung der Schreibszene für das Produkt Text: Wie jeder geschriebene Text für den Historiographen der Schrift seinen Gehalt nicht zuletzt aus dem Prozess seiner Herstellung bezieht, so ist auch der Vorgang des Tätowierens unabhängig von der jeweiligen Motivwahl als Spur eines schmerzhaften Eingriffs in den Körper unmittelbar Bestandteil von deren semantischer Aufladung, gleich ob er als religiöses oder Initiationsritual, als Strafe oder 
als Kommunikationsakt, als Imitatio Christi oder als modische Inszenierung zelebriert wird.

Der europäische Diskurs über die Tätowierung zeichnet sich gegenüber anderen Kulturkreisen dadurch aus, dass diese Semantisierung des Tätowierens von diesem technischen Primärphänomen aus von Anfang an weitgehend über die Auseinandersetzung mit der Schrift bestimmt ist. Das Alte Testament erklärt das Recht auf die Einschreibung von Zeichen in den menschlichen Körpers zum alleinigen Privileg Gottes: Der Brudermörder Kain wird durch das Zeichen, das Gott ihm einträgt, der irdischen Gerichtsbarkeit nicht etwa unterstellt, sondern ausdrücklich entzogen, während die Menschen selbst ,keine Platte machen auf ihrem Haupt noch ihren Bart abscheren und an ihrem Leib kein Mal stechen" ${ }^{\text {" }}$ dürfen. Als Ebenbilder Gottes geschaffen, machen sie sich eines Sakrilegs schuldig, wenn sie das Privileg ihres Schöpfers usurpieren. Auch wenn sich aus diesen Belegstellen nicht.zuverlässig entnehmen lässt, ob die darin genannten Male als Tätowierungen gedacht werden müssen, ist doch der historische Quellgrund vor allem des Kainsmals einer, der diese Vermutung stïtzt: In schriftlichen Zeugnissen seit dem 6. Jahrhundert vor Christus also etwa der Entstehungszeit des Alten Testamentes - belegt, wahrscheinlich aber schon früher, wurden straffällige Sklaven und Kriegsgefangene der Griechen meist im Gesicht, gelegentlich auch auf Armen und Händen mit Schriftzügen tätowiert, die sie allgemein lesbar und vor allem unlöschbar als Besitztum ausstellten. Die römische Kultur weitete diese Technik dann auch auf Soldaten aus, um deren Desertion zu verhindern. Auch literarisch wurde diese Beziehung zwischen Tätowierung und Schrift bereits früh produktiv. Um 250 v. Chr. stellte der griechische Dichter Herondas von Kos eine solche Gesichtstätowierung in den.Mittelpunkt einer seiner Mimiamben, in der eine eifersüchtige Dame.ihrem Sklaven und untreuen Geliebten mit einer Gesichtstấtowierung droht, und im 1 . Jahrhundert n. Chr. setzte Petronius eine solche Tätowierung in seinem Satyricon bereits als Versatzstück seiner Romanpoetologie ein. ${ }^{9}$

Trotz des biblischen Verbots ist auch in der Geschichte des Christentums die Tätowierung als gängige Praxis überliefert. Was mit schlichten Ingroupmarkierungen in Form des Namens Christi, des Fisches oder des Kreuzes begann, an denen die in der Diaspora verstreuten Christen sich ihresgleichen zu erkennen gaben, entfaltete im Lauf der Jahrhunderte - wohl unter dem Einfluss heidnischer Völker wie etwa der britischen Pikten - eine Eigendynamik, aufgrund derer die Tätowierung im 8. Jahrhundert auch in den Bilderstreit hineingezogen wurde; das Konzil in Northumberland sah sich veranlasst, die Tätowierung als Bild zu verbieten, indem es die oben zitierte Passage der Heiligen Schrift eindeutig auf die Tätowierung hin auslegte. Gleichzeitig aber blieb die Tätowierung als Strafpraxis gerade in diesem Kontext erhalten: $\operatorname{Im} 9$. Jahrhundert ließ der ikonoklastische byzantinische Kaiser Theophilos die beiden Brüder Theodorus und Theophanes für das Verbrechen der Bildanbetung 
dazu verurteilen, dass ihnen Schriftzeichen in die Gesichter punktiert wurden. Selbst wenn die anekdotisch anmutende Information, es habe sich dabei um jeweils zwölf Zeilen eines schlechten jambischen Gedichts gehandelt, eine fiktionale Anlagerung wäre, bezeugte sie in sich schon die Wirkungsmacht der Schrifttätowierung. ${ }^{10}$

Unter dem Druck des Bilderverbots auf der einen und der Persistenz der schriftlichen Straftätowierung auf der anderen Seite kam es dann in Mittelalter und früher Neuzeit zu einem eigentümlichen, typisch europäischen Phänomen: Die mittelalterliche Mystik griff die Tätowierung im Kontext jener religiösen Ästhetik der Stigmatisierung auf, mit der der gläubige Körper in der Ekstase der Versenkung sich mit dem Körper des gefolterten Christus identifizierte. Das berühmteste Beispiel dafür ist der Mystiker Heinrich Seuse, der sich zu Beginn des 14. Jahrhunderts. in einer solchen.Ekstase den Namen Christi auf die Haut über seinem Herzen schrieb. Unter anderem an diese Tradition knüpften dann auch die Pilger an, die nach Beendigung der Kreuzzüge nach Jerusalem und anderen heiligen Stätten wallfahrteten. Vor allem in Palästina florierte während der frühen Neuzeit das Geschäft professioneller Tätowierer, die in die Arme ihrer Kunden Abbildungen und Beschreibungen der wichtigsten Jerusalemer Sehenswürdigkeiten eintätowierten, nicht nur, damit diese zu Hause beweisen konnten, dass sie wirklich in Jerusalem gewesen waren, sondern auch als Dokument des an Christi Märtyrium erinnernden Schmerzes, dem sie sich damit unterzogen hatten. ${ }^{11}$

Gleichzeitig begann im 16. Jahrhundert der Influx von Informationen über Tätowierungen von Wilden vor allem Amerikas, Reiseberichte, die Illustrationen tätowierter Indianer zeigten. Theodore de Bry publizierte 1590 in Frankfurt am Main unter dem. Sammeltitel America eine ganze Serie solcher Berichte zusammen mit den in Kupfer gestochenen Abbildungen. Durch die Zusammenstellung dieser Abbildungen verläuft eine eigentümliche Grenze: Während die Bilddarstellungen der amerikanischen Indianer durchweg rein ornamentale Tätowierungen protokollieren, also Punkt-, Linien- und Flächentätowierungen ohne den Repräsentationscharakter von Abbildungen, zeigen einige ebenfalls beigegebene Stiche nach aquarellierten Zeichnungen Jacques de Moynes Pflanzen. So ist eine weibliche Piktin, also eine europäische Ureinwohnerin, mit deutlich erkennbaren Pflanzenmotiven tätowiert - Pflanzen allerdings, die nicht in England wuchsen, sondern in Amerika, und so auf dem Körper des historisch Eigenen eine Repräsentationsökonomie in Szene setzten, die, indem sie auch motivisch auf den Status der Tätowierung als Index des Fremden verwies, selbstreferentiell auf das Wesen künstlerischer Bilddarstellung unter den Bedingungen der europäischen Kultur abhob: „Le Moyne's painting," so fasst Juliet Fleming zusammen, ,reveals itself as the painting not of a woman, but of metaphor itself. Its purpose, in brief, is to evoke admiration for the impossible achievements of art"12 - eine Bewunderung, die, indem sie von der Tätowierung stimuliert wurde, mit den ,impossible achieve- 
ments of art" zugleich auch die Unmöglichkeit eines europäischen tätowierten Körpers außerhalb des Schutzraums künstlerischen Fiktionsprivilegs bestätigte.

Auf der anderen Seite dieser Grenze markierte die fremde Tätowierung des ,Wilden' seine unüberbrückbare Distanz zur europäischen Ökonomie der Repräsentation und begründete damit eine Darstellungskonvention, die sich bis in das 19. Jahrhundert hinein hielt. Die Tafeln, die Wuttke seinem Werk beigibt, zeigen ausschließlich ornamentale Zeichen, auch wenn Wuttke sich alle Mühe gibt, in ihnen immer wieder erkennbare Figuren auszumachen um, wie zu sehen war, ihre Unlesbarkeit konstatieren zu können. So entsteht der - historisch falsche - Eindruck, die Bildtätowierung, wie sie etwa in der Pilgertätowierung erscheint, sei eine europäische Erfindung, die dann nach 1900 in den ganzkörpertätowierten Schaustellern mit ihren Galerien berühmter.Persönlichkeiten .eine spektakuläre Blüte erlebt. Richtig aber dst, dass die Tätowierung in Europa von der Geschichte der Schrift als jener Kulturtechnik aus, die vor allen anderen den zivilisierten Europäer vom unzivilisierten Fremden schied, so konsequent unter dem Aspekt des lesbaren Zeichens rezipiert wurde, dass bereits die frühneuzeitliche Ethnographie die Tätowierung nur als zwar unlesbares, wie die Schrift aber auch dezidiert unfigürliches Notationssystem protokollierte, obwohl, wie sich an zwischen 1100 und 1519 bestatteten tätowierten Mumien der Tolteken und Azteken nachweisen lässt, die figürliche Tätowierung existierte und auch von Eroberungsreisenden gesehen worden sein muss. ${ }^{13}$

Ihren entscheidenden Impuls bekam diese schriftfixierte Rezeptionshaltung am Ende des 18. Jahrhunderts, als James Cook von seiner zweiten Weltreise 1771 das entscheidende Wort mitbrachte, ,ein schönes, geheimnisvolles "Wort, dasisich beinah wie von selbst in die graue Großhirntinde des Europạers "tätowierte und bis heute nicht ausgelöscht ist"14. „Both sexes indent or prick the flesh about and below the hips in a multitude of places, with the points of sharp bones," schreibt Cook in dem noch im Jahr seiner Ankunft in England veröffentlichten Reisebericht über die Eingeborenen von Tahiti, ,and these indentures they fill with a deep blue or blackish paint, which ever after continues, and discolours the skin in those places, rendering it black. This practice is universal among them, and is called tat-tow $[\ldots] . "{ }^{* 15}$

Zusammen mit diesem Wort importierte Cooks Reisebericht eine kleine Geschichte, die Tätowierung und Schrift explizit aufeinander anwies: Der von den Eingeborenen verwendete Begriff ,tat-tow' nämlich, berichtet Cook, sei ,a term which they afterwards applied to letters when they saw us write, being themselves perfectly illiterate. " 16 Die Eingeborenen, ,being [ . . . ] perfectly illiterate," richten den von Cook protokollierten Blick auf die Schrift nicht auf den Inhalt des Geschriebenen, sondern auf den Prozess des Schreibens selbst und identifizieren damit ein Phänomen, das in der europäischen Aufklärung Symptom eines folgenreichen Paradigmenwechsels ist: Schrift und 
Schreiben sind das Instrument, mit dessen Hilfe die Aufklärungspädagogik ihre Anstrengungen vom erzieherischen Zugriff auf den Körper auf denjenigen auf das Innere des Menschen verschiebt; wer liest, bildet den Geist, wer schreibt, kommuniziert vernünftig - aber, und genau dies markiert die Geschichte von den Eingeborenen, auch dem Zugriff auf das Innere des Menschen durch die Schrift eignet eine Gewalt, die, wiewohl sie die Oberfläche des Körpers intakt hält, diesen mit strukturell denselben Zurichtungsspuren zeichnet, wie sie die Tätowierung hinterlässt, vollzieht sich der Prozess des Schreibens doch in einem Bewegungsablauf, dessen Beherrschung sich einer ebenso rigorosen wie irreversiblen Disziplinierung verdankt: „Die primäre ,Gewalt der Schrift', “ so Roger Chartier, ,ist [ . . . ] jene Gewalt, welche die Schrift dem Körper dessen zufügt, der nach den Regeln schreiben will oder muß." ${ }^{\text {17 }}$

Die Episode aus Cooks Reisebericht ist wohl kaum der Anstoß, sicher aber das Symptom eines kulturkritischen Bewusstseins für den Einfluss der Schrift auf den Körper, das in den Jahren nach Cooks Rückkehr am konsequentesten von Goethe buchstäblich auf den Punkt gebracht wurde. Als in seinem 1795/96 erschienenen Roman Wilhelm Meisters Lehrjahre das naturpoetische Kind Mignon daran stirbt, dass die Pädagogen der Turmgesellschaft ihre Androgynie durch einen Einkleidungsakt in die Geschlechtsidentität einer Frau zwingen, und sie dann im Auftrag dieser Pädagogen einbalsamiert wird, kommt es an ihrem offenen Sarg zu einer merkwürdigen Szene: Mignon, so berichtet der Abbé hier, habe sterbend mit „Inbrunst [ . . ] das Bild des Gekreuzigten, das auf ihren zarten Armen mit vielen hundert Punkten sehr zierlich abgebildet steht" geküsst, und entblößt nun vor den Blicken der Zuschauer „ein Kruzifix, von verschiedenen Buchstaben und Zeichen begleitet $[\ldots]$ blaulich auf der weißen Haut" ${ }^{18}{ }^{18}$ Von dieser Tätowierung ist bisher im Roman nie die Rede gewesen, und eine poetologische Analyse von Mignons Geschichte im Licht dieser plötzlich erscheinenden Zeichen führt zu dem Ergebnis, dass Goethe an ihr die Gewalt der Schrift über selbst diesen eigentlich so nachdrücklich gegen die Schriftkultur der Moderne inszenierten Körper ausstellt. ${ }^{19}$

Vor diesem Hintergrund ist Wuttkes Argumentation für die Geburt der Schrift aus dem Geist der Tätowierung als großangelegte Einlösung des Deutungsangebotes zu verstehen, das der Tätowierung mit der Affinitätsunterstellung zwischen ihr und der Schrift in Europa sukzessive angelagert wurde: Vorgeprägt im Brauch der schriftförmigen Tätowierung der griechischen und römischen Antike, der mystischen Identifikation von Schrift und Körper zwischen Mittelalter und früher Neuzeit und der selektiven Wahrnehmung der fremden Tätowierung durch die Ethnographen des Kolonialisierungszeitalters, war diese Affinitätsunterstellung von der modernen Schriftkultur um 1800 als Paradigma der kritischen Reflexion auf die Zurichtung des Körpers durch die Schrift etabliert und von Wuttke schließlich als Basis für die Emergenz eben dieser Kultur ratifiziert worden. 
Wuttkes Geschichtsentwurf steht dabei seinerseits bereits wieder auf einer Schwelle historischen Bewusstseinswandels im europäischen Umgang mit der Tätowierung, die der italienische Gerichtspsychiater Cesare Lombroso vier Jahre nach Erscheinen von Wuttkes Buch mit seiner Schrift L'uomo delinquente programmatisch überschreitet: Wuttkes Postulat vom Willen der Schrift als dem Menschen a priori eingeborenes Humanum erscheint bei Lombroso als Setzung eines kausalen Zusammenhanges zwischen Tätowierung und eingeborener Delinquenz, der die Tätowierung zum Metazeichen für eine atavistische moralische Abnormität erklärt. Lombrosos Buch ist ein Exerzitium in der Tätowierung normativ oktroyierter Lesbarkeit, begleitet von Bilddarstellungen, die dem Leser die einzelnen Tätowierungen in Vokabeln delinquenter Selbstaussagen übersetzen, angefangen vom Totenkopf, der einen Mord, bis zur nackten Frau, die eine Vergewaltigung dokumentiert, gleich ob die solcherart in den Körper niedergelegte.Tat bereits vollzogen ist oder nicht. In Nachfolge Lombrosos geht Adolf Loos 1908 in seinem Essay Ornament und Verbrechen so weit, zu behaupten: „Wenn ein tätowierter in freiheit stirbt, so ist er eben einige jahre, bevor er einen mord verübt hat, gestorben." ${ }^{\star 20}$

Während Wuttke die Tätowierung bei aller rhetorisch-performativen Entstellung des historischen Phänomens doch wesentlich als ein solches Phänomen im Kontext eines spezifischen Entwicklungsprozesses begriffen hatte, ist das, was Lombroso und Loos als Tätowierung verhandeln, Effekt eines Schreibprozesses, der das Instrument Schrift als eine von allen anderen Kommunikationsformen unabhängige Machttechnologie und damit deren Recht an ihrem Gegenstand absolut setzt. Genau hier schließt Kafka 1914 mit seiner Erzählung In der Strafkolonie an, in der eine in Anlehnung an den 1897 patentierten ersten elektrischen Tätowierapparat entwickelte Maschine an dem Delinquenten eine nach zwölf Stunden tödliche Ganzkörpertätowierung vollzieht. Einer Begründung für das Urteil, das dem zu Strafenden hier in Form einer von vielen Zieraten umgebenen Schrift auf den Leib geschrieben wird, bedarf es dabei nicht, denn „die Schuld ist immer zweifellos“21 - wer schreibt, dessen Körper ist in Kafkas Werk immer schon der Gewalt der Schrift verfallen und ist darin in paradoxaler Unausweichlichkeit desto sicherer a priori zum Tode verurteilt, je emphatischer er sich anhand dieser Gewalt gegen sie aufzulehnen versucht.

Es ist sicher kein Zufall, dass die diskursive Koppelung von Tätowierung und Schrift, wie sie hier in Kafkas Erzählung einen letzten Höhepunkt erreicht, in dem Moment an Bedeutung zu verlieren beginnt, in dem die Schriftkultur zunehmend von den neuen Bild- und Tonmedien des 20. Jahrhunderts durchsetzt und entmachtet wird. Ebenso wenig aber ist es ein Zufall, dass diese Koppelung in dem Moment wieder auftaucht, als der technologische Fortschritt dieser Medien den menschlichen Körper żu erfassen und, in den Worten Donna Haraways, als Cyborg zu rekonfigurieren ansetzt: Die „Legung elektromagnetischer Dräthe," auf die Wuttke einst sein Gesamtprojekt 
hin perspektiviert hat, um in euphorischer Gewissheit von der Integrationskraft der Schrift deren „Allgegenwart“ (10) vorauszusagen, hat langfristig die Voraussetzungen für eine Allgegenwart des Bildes geschaffen, die nicht mehr wie die Telegraphie das geschriebene Zeichen, sondern den Körper zu dematerialisieren beginnt.

Und so ist es denn auch der Körper, an dem die Affinität von Schrift und Tätowierung nun gegen die Schrift auf dem Körper-Bild insistiert: Seit den 1980er Jahren betreiben immer mehr Tätowierer in Amerika die Tätowierung gegen ihre modisch-populäre Trivialisierung als bildkünstlerische Form, die auf den je tätowierten Körper hin entwickelt wird und damit auch immer Unikat ist. Arbeiten wie die der in New Mexico tätigen Künstlerin Cynthia Whitkin subvertieren die Affinitätsunterstellung, die das Verhältnis zwischen Tätowierung und Schrift zugunsten der letzteren hierarchisiert hatte, indem sie anhand der.Juxtaposition von:weder figürlich nọ schriftförmig gestalteten Tätowierungen und Titelzuschreibungen die Unlesbarkeit, auf der diese Hierarchisierung aufruhte, vom Körper aus gegen die Schrift wenden. So fasst der Titel einer von ihr hergestellten, in unregelmäßig gezackten Linien verlaufenden Schultertätowierung „Automatic Writing“ die Rezeptionsgeschichte der Tätowierung in einer knappen ironischen Pointe zusammen: Einerseits der Name eines Verfahrens, mit dem die surrealistische Kunstbewegung die Schreibszene des 20. Jahrhunderts durch das automatische als intuitives, nicht rational gerastertes Schreiben radikal zu überschreiten suchte, rekapituliert er zugleich die Bewegung, mit der der abendländische Diskurs über die Tätowierung den Automatismus ihrer Verbindung mit der Schrift erzeugt - und so bereits in Wuttkes Geschichte der Schrift eine Dynamik eingetragen hatte, die, aus der Perspektive auf diese Bewegung gesehen, metaphorisch den Namen des „Automatic Writing“" zu tragen verdient.

${ }^{1}$ Heinrich Wuttke, Geschichte der Schrift und des Schrifttums von den rohen Anfängen des Schreibens in der Tatuirung bis zur Legung elektromagnetischer Dräthe. Bd. 1: Die Entstehung der Schrift, die verschiedenen Schriftsysteme und das Schrifttum der nicht alfabetarisch schreibenden Völker (Leipzig: Fleischer, 1872), im folgenden mit unmittelbar im Text angeschlossener Seitenangabe zitiert. Hier: $79 f$. $569-572$.

${ }_{2}^{2}$ Allgemeine Deutsche Biographie (Leipzig: Duncker und Humblot, 1898), Bd. 444,

${ }^{3}$ Adam Johann von Krusenstern, Reise um die Welt in den Jahren 1803, 1804, 1805 und 1806 (St. Petersburg 1814). Hg. P. Werner Lange (Köln/Wien: Böhlau, 1986).

${ }^{4}$ Zum Begriff der "Schreibszene" vgl. Rüdiger Campe, „Die Schreibszene. Schreiben," Paradoxien. Dissonanzen. Zusammenbrüche. Situationen offener Epistemologie. Eds. Hans Ulrich Gumbrecht und K. Ludwig Pfeiffer (Frankfurt am Main: Suhrkamp, 1991) 759-772, sowie die Arbeit des von Martin Stingelin geleiteten Forschungsprojekts „Zur Genealogie des Schreibens" in Basel, die erst vor kurzen einen Band über die Auswirkungen u.a. der weiter unten von Wuttke erwähnten "Schreibkugel“" auf die zeitgenössische Geschichte des Schreibens präsentiert hat: „Schreibkugel ist ein Ding gleich mir: von Eisen “. Schreibszenen im Zeitalter der Typoskripte. Eds. Davide Giuriato, Martin Stingelin, Sandro Zanetti (München: Fink, 2005) [Zur Genealogie des Schreibens 2]. 
${ }^{5} \mathrm{Zu}$ diesem Begriff vgl. Friedrich Kittler, Aufschreibesysteme 1800-1900 (München: Fink, 1987).

${ }^{6}$ Vgl. dazu Elain Scarry, The Body in Pain. The Making and Unmaking of the World (New York / Oxford: Oxford UP, 1985), sowie Schmerz und Erinnerung. Ed. Roland Borgards (München: Fink, 2005).

${ }^{7}$ Alfred Gell, Wrapping in Images. Tattooing in Polynesia (Oxford: Oxford UP, 1993) [Oxford Studies in Social and Cultural Anthropology; Cultural Forms] 9.

83. Mose 21, 5.

${ }^{9}$ Vgl. dazu C. P. Jones, ,Stigma: Tattooing and Branding in Graeco-Roman Antiquity," The Journal of Roman Studies 77 (1987) 139-155; vgl. auch ders. „Stigma and Tattoo," Written on the Body. The Tattoo in American and European History. Ed. Jane Caplan (Princeton: Princeton UP, 2000) 1-16, sowie Mark Gustafson, „Inscripa in fronte: Penal Tattooing in Late Antiquity," Classical Antiquity 16:2 (1997), 79-104; vgl. auch ders., " The Tattoo in the Later Roman Empire and Beyond," Written on the Body 17-31.

${ }^{10} \mathrm{Vgl}$. Gustafson, ,Inscripta in fronte" 20.

${ }^{1}$ Vgl. Ulrike Landfester, "Gestochen scharf. Die Tätowierung als Erinnerungsfigur," Schmerz und Erinnerung 83-98, sowie Mordechai Lewy, ,Jerusalem unter der Haut. Zur Geschichte der JerusäJemer Pilgertätowierung," Zeitschrift für Religions- und Geistesgeschichte 55:1 (2003) 1-39.

${ }^{12}$ Juliet Fleming. „The Renaissance Tattoo," Written on the Body 61-82. Hier: 75.

${ }^{13}$ Vgl. dazu Wilfred Dyson Hambly, The History of Tattooing and its Significance (London: Witherby, 1925) 808-835.

${ }^{14}$ Stephan Oettermann, Zeichen auf der Haut. Die Geschichte der Tätowierung in Europa (Frankfurt am Main: Syndikat, 1985) 9.

${ }^{15}$ [James Cook], A Journal of a Voyage round the World, In His Majesty's Ship Endeavour, In the Years 1768, 1769, 1770, and 1771; Untertaken in Pursuit of Natural Knowledge, at the Desire of the Royal Society: Containing All the various Occurrences of the Voyage. [... I To which ist added, A Concise Vocabulary Of the Language of Otahitee (London 1771) Nachdruck (Amsterdam / New York: P. Schippers, 1967) [Bibliotheca Australia 14] 44.

${ }^{16} \mathrm{Ebd}$.

${ }^{17}$ Roger Chartier, ,Macht der Schrift, Macht über die Schrift“ Schrift. Eds. Hans Ulrich Gumbrecht, K. Ludwig Pfeiffer (München: Fink, 1993) [Materialität der Zeichen Reihe A 12] 147-156. Hier: 154.

${ }^{18}$ Johann Wolfgang Goethe, „Wilhelm Meisters Lehrjahre. Ein Roman." Sämtliche Werke nach Epochen seines Schaffens. Münchner Ausgabe. Ed. Karl Richter in Zusammenarbeit mit Herbert G. Gônfert, Norbert Miller, Gerhard Sauder und Edith Zehm (München: Hanser, 1988), Bd. 5, 578 .

${ }^{19}$ Vgl. dazu ausführlich Ulrike Landfester, „Pathographien des Schreibens. Zur poetologischen Funktion von Tätowierungen in Johann Wolfgang von Goethes, Wilhelm Meisters Lehrjahre' und Franz Kafkas ,In der Strafkolonie’"“ Poetica 33: 1-2 (2002) 159-189.

${ }^{20}$ Adolf Loos, „Ornament und Verbrechen,“ Adolf Loos, Trotzdem. 1900-1930 (Innsbruck: Brenner Verlag, 1931) 79-92. Hier: 79f.

${ }^{21}$ Franz Kafka, „In der Strafkolonie,“ Schriften, Tagebücher, Briefe. Kritische Ausgabe. Ed. Jürgen Born, Gerhard Neumann, Malcolm Pasley, Jost Schillemeit. [Ohne Bandnummerierung:] Drucke zu Lebzeiten. Ed. Wolf Kittler, Hans-Gerd Koch, Gerhard Neumann (Frankfurt am Main: Fischer, 1994) 201-248. Hier: 212. 